\title{
A POTENTIAL THEORY FOR MONOTONE MULTIVALUED OPERATORS
}

\author{
BY
}

\author{
G. ROMANO, L. ROSATI, F. MAROTTI DE SCIARRA, AND P. BISEGNA
}

Istituto di Scienza delle Costruzioni, Facoltà di Ingegneria, Università di Napoli "Federico II"

\begin{abstract}
The concept of cyclic monotonicity of a multivalued map has been introduced by R. T. Rockafellar with reference to the subdifferential operator of a convex functional. He observed that cyclic monotonicity could be viewed heuristically as a discrete substitute for the classical condition of conservativity, i.e., the vanishing of all the circuital integrals of a vector field. In the present paper a potential theory for monotone multivalued operators is developed, and, in this context, an answer to Rockafellar's conjecture is provided. It is first proved that the integral of a monotone multivalued map along lines and polylines can be properly defined. This result allows us to introduce the concept of conservativity of a monotone multivalued map and to state its relation with cyclic monotonicity. Further, as a generalization of a classical result of integral calculus, it is proved that the potential of the subdifferential of a convex functional coincides, to within an additive constant, with the restriction of the functional on the domain of its subdifferential map. It is then shown that any conservative monotone graph admits a pair of proper convex potentials which meet a complementarity relation. Finally, sufficient conditions are given under which the complementary and the Fenchel's conjugate of the potential associated with a conservative maximal monotone graph do coincide.
\end{abstract}

1. Introduction. Potential theory for nonlinear operators on topological vector spaces is a well-established branch of functional analysis; an exhaustive and detailed presentation can be found in the book by Vainberg [13].

The classical theory is concerned with the problem of finding a real-valued functional whose gradient is an assigned operator and with the conditions ensuring that such a functional does in fact exist.

The well-known general condition turns out to be the conservativity of the operator, that is, the vanishing of the related circuital integral along every closed curve in the domain of the operator.

In convex analysis the subdifferential operator of a (not necessarily differentiable) proper convex lower semicontinuous functional is defined to be the multivalued operator that maps each point in the domain of the functional to the closed convex set of its subgradients.

Received May 31, 1991.

1991 Mathematics Subject Classification. Primary 31-02. 
It is then quite natural to put the following question: which conditions must be fulfilled by a multivalued operator in order that it be the subdifferential operator of a proper convex lower semicontinuous functional?

A complete answer was given by Rockafellar in $[9,11]$ : the necessary and sufficient property to be satisfied by the multivalued operator is that of maximal cyclic monotonicity.

Rockafellar's definitions and arguments have been reproduced in [8] by Moreau who attributes to Minty [7] the first appeal to maximal monotonicity of a multivalued operator.

In February 1991, while giving a lesson on nonsmooth mechanics, the first author was asked by a doctoral student about the physical meaning of the cyclic monotonicity of a multivalued operator.

This quite natural question drew the authors' attention to the connection between cyclic monotonicity and conservativity.

It soon became apparent that a satisfactory answer to the question required the development of a potential theory for monotone multivalued operators.

The first step in this direction was then to give a definite meaning to the concept of the integral of such operators.

To hit the target it was first noticed that the integral of a real monotone multivalued function on a bounded interval is well defined since the monotonicity of the function implies that its "jumps" cannot be too many, in the sense that the set of all "jumps" can be at most a countable one.

Starting from this basic idea, the definition of the line integral of a monotone multivalued operator is straightforward and immediately extendible to integrals along polylines. Such definition allows us to introduce the concept of conservativity of a monotone multivalued operator.

With these tools at hand, the relation between conservativity and cyclic monotonicity can be established and the potential of a conservative monotone multivalued map can be defined by an integral formula, in perfect analogy with the classical theory.

In particular, it is shown that the potentials of the right and the left maps associated with a conservative monotone graph are proper convex functionals that meet a complementarity property.

It is further proved that the complementary and the Fenchel's conjugate of the potential associated with a conservative maximal monotone graph do coincide under suitable regularity assumptions.

The concept of the potential of a monotone multivalued operator provides an effective tool for the systematic search of variational principles associated with problems stated in terms of multivalued operators.

Applications to nonsmooth problems of structural mechanics will be developed in forthcoming papers.

2. Some definitions of convex analysis. Let us recall here some basic definitions and properties of convex analysis, which we shall refer to in the sequel. A comprehensive treatment of the subject can be found, for example, in $[1,3,8,12]$.

Let $\left(X, X^{\prime}\right)$ be an ordered pair of dual locally convex topological vector spaces 
and $\langle\cdot, \cdot\rangle$ the related compatible duality pairing mapping the product space $X \times X^{\prime}$ into the set of reals $\Re$.

Given a proper convex functional $f: X \mapsto \Re \cup\{+\infty\}$, its effective domain is the nonempty convex set on which it assumes finite values

$$
\operatorname{dom} f=\{x \in X \mid f(x)<+\infty\} .
$$

The Fenchel's conjugate $f^{*}: X^{\prime} \mapsto \Re \cup\{+\infty\}$ of $f$ is defined as

$$
f^{*}\left(x^{*}\right)=\sup _{y \in X}\left\{\left\langle x^{*}, y\right\rangle-f(y)\right\}
$$

so that the following Fenchel's inequality holds:

$$
f(y)+f^{*}\left(x^{*}\right) \geq\left\langle x^{*}, y\right\rangle \quad \forall y \in X, \forall x^{*} \in X^{\prime} .
$$

The pairs $\left(x, x^{*}\right)$ for which Fenchel's inequality holds as an equality are said to be conjugate and are related by the subdifferential multivalued operator $\partial$, defined by

$$
x^{*} \in \partial f(x) \stackrel{\text { def }}{\Longleftrightarrow} f(z)-f(x) \geq\left\langle x^{*}, z-x\right\rangle \quad \forall z \in X .
$$

If the closed convex subdifferential set $\partial f(x)$ is nonempty, the functional $f$ is said to be subdifferentiable at $x$ and each $x^{*} \in \partial f(x)$ is called a subgradient of $f$ at $x$.

For any convex functional $f$, it turns out to be

$$
x^{*} \in \partial f(x) \Rightarrow x \in \partial f^{*}\left(x^{*}\right),
$$

and the inverse implication holds if

$$
\liminf _{z \rightarrow x} f(z) \geq f(x) \quad \forall x \in X,
$$

that is, if $f$ is lower-semicontinuous (1.s.c.) [3].

3. Integrals of monotone multivalued maps. A graph $G$ is a nonempty subset of the product space: $G \subseteq X \times X^{\prime}$. Two multivalued maps are naturally associated with a graph $G$ :

$$
\begin{aligned}
\text { the right map } & \vec{G}: X \mapsto X^{\prime}, \\
\text { the left map } & \stackrel{\leftarrow}{G}: X^{\prime} \mapsto X,
\end{aligned}
$$

defined by

$$
\begin{aligned}
\vec{G}(x) & =\left\{x^{*} \in X^{\prime} \mid\left(x, x^{*}\right) \in G\right\} \subseteq X^{\prime}, \\
\overleftarrow{G}\left(x^{*}\right) & =\left\{x \in X \mid\left(x, x^{*}\right) \in G\right\} \subseteq X,
\end{aligned}
$$

so that $\vec{G}(\overleftarrow{G})$ is a correspondence between elements of $X\left(X^{\prime}\right)$ and subsets of $X^{\prime}(X)$.

We shall write graph $(\vec{G})$ or graph $(\overleftarrow{G})$ to denote $G$ in terms of the associated maps. 
The domain and the range of the right and left map,

$$
\begin{aligned}
& \operatorname{dom} \vec{G}=\text { range } \stackrel{\leftarrow}{G} \stackrel{\text { def }}{=}\{x \in X \mid \vec{G}(x) \neq \varnothing\}, \\
& \operatorname{dom} \stackrel{\leftarrow}{G}=\operatorname{range} \overleftrightarrow{G} \stackrel{\text { def }}{=}\left\{x^{*} \in X^{\prime} \mid \stackrel{\leftarrow}{G}\left(x^{*}\right) \neq \varnothing\right\},
\end{aligned}
$$

are the projections of $G$ on $X$ and $X^{\prime}$ respectively.

The product sets $\operatorname{dom} \vec{G} \times X^{\prime}$ and $X \times \operatorname{dom} \overleftarrow{G}$ are then subsets of the space $X \times X^{\prime}$, which will respectively be referred to as the vertical strip and the horizontal strip of $G$ and denoted by v-strip $(G)$ and h-strip $(G)$.

The set $\operatorname{dom} \vec{G} \times \operatorname{dom} \stackrel{\leftarrow}{G}=$ v-strip $(G) \cap$ h-strip $(G)$ will be called the frame of $G$ and denoted by frame $(G)$.

Definition 3.1. Monotonicity. A graph $G \subseteq X \times X^{\prime}$ is said to be monotone if

$$
\left\langle x_{2}^{*}-x_{1}^{*}, x_{2}-x_{1}\right\rangle \geq 0 \quad \forall\left(x_{i}, x_{i}^{*}\right) \in G, i=1,2 .
$$

A multivalued map $M: X \mapsto X^{\prime}$ will be said to be monotone if $\operatorname{graph}(M)$ is monotone.

Monotone graphs that meet a maximality property do play a special role in the subsequent developments; we need then the following:

Definition 3.2. Maximal Monotonicity. A graph $G_{\text {ex }} \subseteq X \times X^{\prime}$ is said to be an extension of a graph $G \subseteq X \times X^{\prime}$ if $G \subseteq G_{\text {ex }}$. Whenever $G$ and $G_{\text {ex }}$ are monotone, $G_{\text {ex }}$ will be called a monotone extension of $G$. It will be called a proper monotone extension of $G$ if $G_{\text {ex }} \neq G$.

Given a set $S \subseteq X \times X^{\prime}$, a monotone graph $G \subseteq S$ is said to be maximal in $S$ if it cannot be properly extended to a monotone graph $G_{\mathrm{ex}} \subseteq S$. A monotone graph $G$ is said to be maximal if it is maximal in $X \times X^{\prime}$. The existence of a maximal monotone extension of a given graph is ensured by Zorn's Lemma. Maximal monotonicity can be equivalently stated as $[8,11]$.

$$
\left\langle z^{*}-x^{*}, z-x\right\rangle \geq 0 \quad \forall\left(z, z^{*}\right) \in G \Leftrightarrow\left(x, x^{*}\right) \in G .
$$

The maps $\vec{G}$ and $\overleftarrow{G}$ themselves are then said to be maximal monotone.

Monotone extensions of a monotone graph in a one-dimensional case are sketched in Fig. 1.

The next statement yields a useful result concerning the images of the multivalued maps associated with a maximal monotone graph.

Lemma 3.3. Closed convex images. Let $G \subseteq X \times X^{\prime}$ be a maximal monotone graph. Then

$$
\begin{gathered}
\vec{G}(x) \subseteq X^{\prime} \text { is a closed convex set } \forall x \in \operatorname{dom} \vec{G}, \\
\stackrel{\leftarrow}{G}\left(x^{*}\right) \subseteq X \text { is a closed convex set } \forall x^{*} \in \operatorname{dom} \overleftrightarrow{G} .
\end{gathered}
$$

Proof. By definition of maximal monotone graph, the set

$$
\vec{G}(x)=\left\{x^{*} \in X^{\prime} \mid\left(x, x^{*}\right) \in G\right\}=\left\{x^{*} \in X^{\prime} \mid\left\langle z^{*}-x^{*}, z-x\right\rangle \geq 0 \forall\left(z, z^{*}\right) \in G\right\},
$$




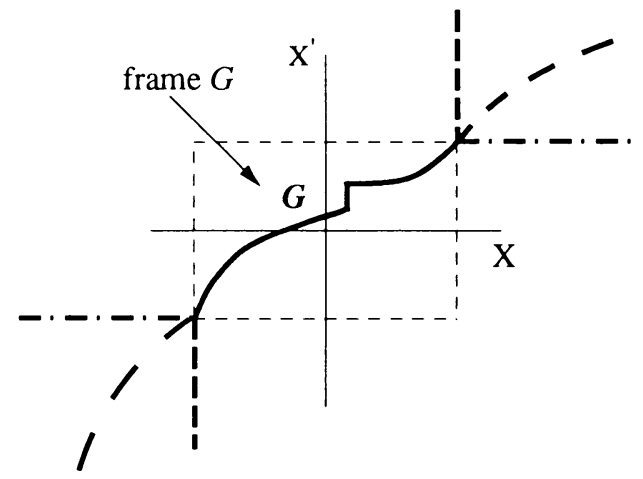

FIG. 1. Monotone extensions of a monotone graph $G$.

as intersection of a family of closed half-spaces, turns out to be closed and convex. A symmetric argument yields the analogous result for the map $\overleftarrow{G}$.

In order to develop a potential theory for monotone multivalued maps, it is fundamental to prove that the line integral of these maps can be unambiguously defined. To this end the following preliminary results are needed.

Definition 3.4. Single-valued representatives. Let us consider a real monotone multivalued map $\Phi(t)$ with $\operatorname{dom} \Phi=[0,1]$ and its maximal monotone extension in $[0,1] \times \Re$, which is

$$
\Phi_{m}(t)=\left[\Phi^{-}(t), \Phi^{+}(t)\right]
$$

where

$$
\begin{aligned}
& \Phi^{-}(t) \stackrel{\text { def }}{=} \sup \{\bigcup \Phi(\tau) \mid \tau<t\} \in \Re \cup\{-\infty\}, \\
& \Phi^{+}(t) \stackrel{\text { def }}{=} \inf \{\bigcup \Phi(\tau) \mid \tau>t\} \in \Re \cup\{+\infty\},
\end{aligned}
$$

under the usual convention that the "sup" and the "inf" of the empty set respectively are equal to $-\infty$ and $+\infty$. A real function $\alpha$ from $[0,1]$ into $\Re$ such that

$$
\alpha(t) \in \Phi_{m}(t) \quad \forall t \in[0,1]
$$

will be called a single-valued representative of $\Phi_{m}(t)$, and we shall write $\alpha \in \Phi_{m}$. It is evident that every $\alpha \in \Phi_{m}$ is a monotone function.

In Fig. 2 (see p. 618) a real multivalued map (a) and its maximal monotone extension in the v-strip (b) are illustrated.

REMARK 3.5. It can be proved that the subset

$$
S=\left\{t \in[0,1] \mid \inf \Phi_{m}(t)<\sup \Phi_{m}(t)\right\}
$$

on which the multivalued map $\Phi_{m}$ is not single-valued turns out to be finite or countable [2] and coincides with the set of discontinuity points of all its single-valued representatives.

LEMMA 3.6. Integrability of monotone multivalued real maps. Given a real monotone multivalued map $\Phi$ with $\operatorname{dom} \Phi=[0,1]$, its integral on the interval $[0,1]$ is finite and well defined by the formula

$$
\int_{0}^{1} \Phi(t) d t \stackrel{\text { def }}{=} \int_{0}^{1} \Phi_{m}(t) d t \stackrel{\text { def }}{=} \int_{0}^{1} \alpha(t) d t \quad \forall \alpha \in \Phi_{m} .
$$




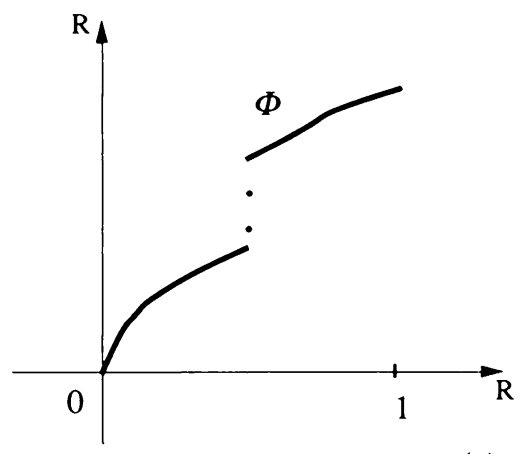

(a)

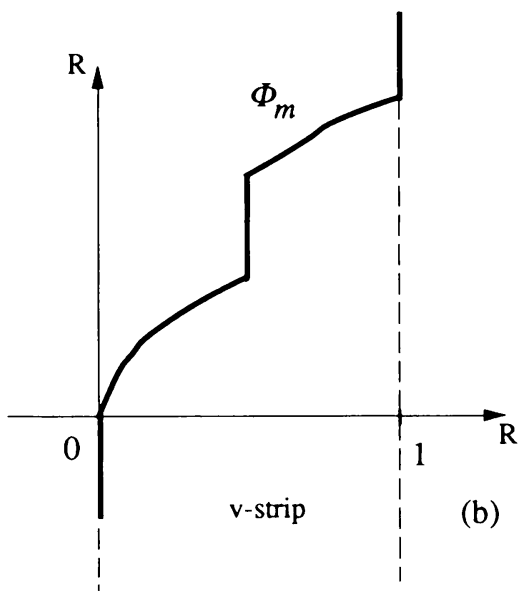

FIG. 2. A monotone graph and its maximal monotone extension in the v-strip.

Proof. Since the set $S$ of discontinuity points of the single-valued representatives $\alpha \in \Phi_{m}$ has a zero Lebesgue measure in $\Re$, it follows that such representatives are continuous Lebesgue almost everywhere (a.e.) on $[0,1]$. Every $\alpha \in \Phi_{m}$ is monotone and finite at the end points, hence bounded on $[0,1]$; a theorem by Vitali-Lebesgue [6] ensures then its Riemann integrability. The value of the integral does not depend on the choice of $\alpha \in \Phi_{m}$, the difference between any two functions $\alpha$ being zero Lebesgue a.e. on $[0,1]$. Finally from the inequalities

$$
-\infty<\sup \Phi(0) \leq \int_{0}^{1} \alpha(t) d t \leq \inf \Phi(1)<+\infty,
$$

it follows that the integral has a finite value.

REMARK 3.7. By virtue of the monotonicity of the functions $\alpha$, the following formula holds for the integral:

$$
\sup \left\{\sum_{i=0}^{n-1} \alpha\left(t_{i}\right)\left(t_{i+1}-t_{i}\right)\right\}=\int_{0}^{1} \Phi(t) d t=\inf \left\{\sum_{i=0}^{n-1} \alpha\left(t_{i+1}\right)\left(t_{i+1}-t_{i}\right)\right\},
$$


where the $\sum$ 's refer to arbitrary partitions of the interval $[0,1]$.

LEMMA 3.8. Monotonicity of the projection along a line segment. Let $M: X \mapsto X^{\prime}$ be a monotone multivalued map, and consider an oriented line segment $[a, b] \subseteq \operatorname{dom} M$ with parametric representation $\hat{x}(t)=a+t h$, where $h=b-a$ and $0 \leq t \leq 1$. The projection of the map $M$ along the segment, which is the real multivalued map

$$
\Psi(t)=\langle M(\hat{x}(t)), h\rangle \stackrel{\text { def }}{=}\left\{\left\langle\hat{x}^{*}(t), h\right\rangle \mid \hat{x}^{*}(t) \in M(\hat{x}(t))\right\},
$$

from $[0,1]$ into $\Re$, has a monotone graph.

Proof. For all $\psi_{1} \in \Psi\left(t_{1}\right), \psi_{2} \in \Psi\left(t_{2}\right)$ we have, by the monotonicity of $M$,

$$
\begin{aligned}
\left(\psi_{2}-\psi_{1}\right)\left(t_{2}-t_{1}\right) & =\left\langle\hat{x}^{*}\left(t_{2}\right)-\hat{x}^{*}\left(t_{1}\right), t_{2} h-t_{1} h\right\rangle \\
& =\left\langle\hat{x}^{*}\left(t_{2}\right)-\hat{x}^{*}\left(t_{1}\right), \hat{x}\left(t_{2}\right)-\hat{x}\left(t_{1}\right)\right\rangle \geq 0,
\end{aligned}
$$

which was to be proved.

It is easy to show that, if $\operatorname{dom} M$ is convex, the monotonicity of the projection of $M$ along every line segment in $\operatorname{dom} M$ implies the monotonicity of the map $M$.

We can now set the definition of line integral for a monotone multivalued map.

Definition 3.9. Line integrals. Let $M: X \mapsto X^{\prime}$ be a monotone multivalued map and $[a, b]$ an oriented line segment in $\operatorname{dom} M$ with parametric representation $\hat{x}(t)=a+t h$, where $h=b-a$ and $0 \leq t \leq 1$. Then we set

$$
\int_{a}^{b}\langle M(x), d x\rangle \stackrel{\text { def }}{=} \int_{0}^{1}\langle M(\hat{x}(t)), h\rangle d t
$$

where the integral is well defined by virtue of Lemmas 3.6 and 3.8. The integral of $M$ along an oriented polyline $\pi$ in $\operatorname{dom} M$ is accordingly defined as the sum of the line integrals along each side.

REMARK 3.10. From the inequality in Lemma 3.6 and the definition of $\Psi(t)$ we get

$$
\left\langle a^{*}, b-a\right\rangle \leq \int_{a}^{b}\langle M(x), d x\rangle \leq\left\langle b^{*}, b-a\right\rangle,
$$

for every line segment $[a, b] \subseteq \operatorname{dom} M$, for all $a^{*} \in M(a)$ and $b^{*} \in M(b)$.

DEFINITION 3.11. An arbitrary subdivision of a segment $[a, b]$ by means of a finite family of segments oriented congruently to $[a, b]$ will be called a refinement of $[a, b]$.

Hence a refinement of a given polyline $\pi$ is any polyline that can be obtained by a suitable refinement of each side of $\pi$.

An immediate consequence of Remark 3.7 is then the following:

THEOREM 3.12. Polyline integrals. Let $M: X \mapsto X^{\prime}$ be a monotone multivalued map. If $\pi$ is any oriented polyline in $\operatorname{dom} M$ we have

$$
\sup \left\{\sum_{i=0}^{n-1}\left\langle x_{i}^{*}, x_{i+1}-x_{i}\right\rangle\right\}=\int_{\pi}\langle M(x), d x\rangle=\inf \left\{\sum_{i=0}^{n-1}\left\langle x_{i+1}^{*}, x_{i+1}-x_{i}\right\rangle\right\},
$$

where the $\sum$ 's refer to arbitrary refinements of $\pi$, the choice of $x_{i}^{*} \in M\left(x_{i}\right)$ being inessential. 
REMARK 3.13. Let $M: X \mapsto X^{\prime}$ be a monotone multivalued map, and let $\widetilde{M}$ be a monotone extension of $M$. If $\pi$ is an arbitrary oriented polyline in $\operatorname{dom} M$, we have

$$
\int_{\pi}\langle\widetilde{M}(x), d x\rangle=\int_{\pi}\langle M(x), d x\rangle .
$$

In fact, the projection of $\widetilde{M}$ along any oriented line segment that belongs to $\pi$ is a monotone extension of the projection of $M$, and then, by virtue of Lemma 3.6, the line integrals of $M$ and $\widetilde{M}$ coincide as well as the integrals along the polyline $\pi$.

Let us now set the definition of curvilinear integral.

Definition 3.14. Curvilinear integrals. Let $M: X \mapsto X^{\prime}$ be a monotone multivalued map with a convex domain, $\omega$ an oriented curve defined by a continuous mapping from $[0,1]$ into $\operatorname{dom} M$, and $\Pi_{\omega}$ the family of iso-oriented polylines whose vertices $x_{i}, i=1, \ldots, n$, belong to $\omega$ such that $x_{1}=\omega(0)$ and $x_{n}=\omega(1)$. The family $\Pi_{\omega}$ can be structured as a directed set by defining in it a partial ordering, denoted by $\prec$, according to which $\pi_{1}, \pi_{2} \in \Pi_{\omega}$ are such that $\pi_{1} \prec \pi_{2}$ if every vertex of $\pi_{1}$ is also a vertex of $\pi_{2}$.

Given the real-valued function $f: \Pi_{\omega} \mapsto \Re$, it is said that $f$ has a generalized limit $f_{\omega}$ through $\Pi_{\omega}[14]$ if

$$
\forall \varepsilon>0 \exists \pi_{\varepsilon} \in \Pi_{\omega}: \pi_{\varepsilon} \prec \pi \in \Pi_{\omega} \Rightarrow\left|f_{\omega}-f(\pi)\right|<\varepsilon,
$$

and we write

$$
\lim _{\pi \in \Pi_{\omega}} f(\pi)=f_{\omega}
$$

The curvlinear integral of the map $M$ along $\omega$ is accordingly defined as

$$
\int_{\omega}\langle M(x), d x\rangle \stackrel{\text { def }}{=} \lim _{\pi \in \Pi_{\omega}} \int_{\pi}\langle M(x), d x\rangle .
$$

The assessment of suitable regularity assumptions on the curve $\omega$, able to ensure the existence of the generalized limit above for any monotone multivalued map, is still an open problem; a result due to Rockafellar concerned with the locally boundedness of a monotone operator along a closed curve in the interior of its domain [10] could be a starting point to develop an existence theorem.

4. Potential theory. At this stage of the analysis we have properly defined the concepts of line and polyline integrals of a monotone multivalued map and depicted their properties.

The next step will consist in the formulation of the potential theory for such maps; to this end we start giving the definition of conservativity, which is fundamental for the subsequent developments of the theory.

Definition 4.1. Conservative multivalued maps. A monotone multivalued map $M: X \mapsto X^{\prime}$ is said to be conservative if

$$
\oint_{\pi}\langle M(x), d x\rangle=0,
$$

for every closed polyline $\pi \subseteq \operatorname{dom} M$. 
If $\operatorname{dom} M$ is convex, the conservativity of $M$ implies, according to Definition 3.14 , that the curvilinear integral along a closed curve $\omega \subseteq \operatorname{dom} M$ exists and vanishes.

Remark 4.2. Conservativity of the extension. If $M: X \mapsto X^{\prime}$ is a conservative monotone multivalued map and $\widetilde{M}$ is a monotone extension of $M$ such that $\operatorname{dom} \widetilde{M}=\operatorname{dom} M$, then $\widetilde{M}$ is conservative. In fact for every closed polyline $\pi \subseteq$ $\operatorname{dom} M$ we get

$$
\oint_{\pi}\langle\widetilde{M}(x), d x\rangle=\oint_{\pi}\langle M(x), d x\rangle=0
$$

by Remark 3.13 and the conservativity of $M$.

The following statement extends a classical result of potential theory to conservative monotone multivalued maps.

Remark 4.3. Path independence. The polyline integral of a conservative monotone multivalued map $M$ along any oriented polyline in its domain connecting two arbitrary points depends only upon the end points of the polyline. If $\operatorname{dom} M$ is convex, the curvilinear integral along any oriented curve $\omega \subseteq \operatorname{dom} M$ exists and depends only upon the end points of the curve.

Definition 4.4. Cyclic monotonicity. A multivalued map $M: X \mapsto X^{\prime}$ satisfying the inequality

$$
\sum_{i=0}^{n}\left\langle x_{i}^{*}, x_{i+1}-x_{i}\right\rangle \leq 0, \quad \text { with } n+1 \equiv 0,
$$

for every $x_{0}, \ldots, x_{n}$ belonging to $\operatorname{dom} M$ and for every choice of $x_{i}^{*} \in M\left(x_{i}\right)$, has been termed cyclically monotone by Rockafellar [9, 11, 12].

Trivially cyclic monotonicity implies monotonicity.

Rockafellar proved that the graph of a multivalued map is included in the graph of the subdifferential of a lower semicontinuous proper convex functional if and only if it is cyclically monotone (see also Moreau [8]).

REMARK 4.5. Let $G \subseteq X \times X^{\prime}$ be a graph. It is easy to prove that the proposition

$$
\sum_{i=0}^{n}\left\langle x_{i}^{*}, x_{i+1}-x_{i}\right\rangle \leq 0
$$

for every choice of $x_{0}, \ldots, x_{n}, x_{n+1}=x_{0}$ belonging to $\operatorname{dom} \vec{G}$ and for every choice of $x_{i}^{*} \in \vec{G}\left(x_{i}\right)$, is equivalent to

$$
\sum_{i=0}^{n}\left\langle x_{i+1}^{*}, x_{i+1}-x_{i}\right\rangle \geq 0
$$

for every choice of $x_{0}^{*}, \ldots, x_{n}^{*}, x_{n+1}^{*}=x_{0}^{*}$ belonging to dom $\overleftarrow{G}$ and for every choice of $x_{i} \in \overleftarrow{G}\left(x_{i}^{*}\right)$. In fact, the two formulas can be obtained by applying the same property to an $n$-tuple $\left(x_{i}, x_{i}^{*}\right) \in G$ with $i=0, \ldots, n$ according to reverse numerations. A simple algebra further shows that

$$
\sum_{i=0}^{n}\left\langle x_{i}^{*}, x_{i+1}-x_{i}\right\rangle=-\sum_{i=0}^{n}\left\langle x_{i+1}^{*}-x_{i}^{*}, x_{i+1}\right\rangle
$$


and

$$
\sum_{i=0}^{n}\left\langle x_{i+1}^{*}, x_{i+1}-x_{i}\right\rangle=-\sum_{i=0}^{n}\left\langle x_{i+1}^{*}-x_{i}^{*}, x_{i}\right\rangle
$$

for every $n$-tuple of points $\left(x_{i}, x_{i}^{*}\right) \in X \times X^{\prime}$. We may thus infer that

$$
\vec{G} \text { cyclically monotone } \Leftrightarrow \overleftarrow{G} \text { cyclically monotone. }
$$

Cyclic monotonicity is then a characteristic property of graph $G$, with the dual spaces $X$ and $X^{\prime}$ playing a symmetric role.

The next theorem shows the relation between the concepts of cyclic monotonicity and conservativity for a monotone map.

THEOREM 4.6. On the relation between cyclic monotonicity and conservativity. A cyclically monotone multivalued map $M: X \mapsto X^{\prime}$ is monotone and conservative. Conversely, if a map $M$ is monotone and conservative with a convex domain, it is cyclically monotone.

Proof. Let $M$ be cyclically monotone. The inequalities in Remark 4.5 imply that, in the formula stated in Theorem 3.12, the "sup" is nonpositive and the "inf" is nonnegative. It follows that all three terms must vanish and hence $M$ is conservative.

Now let $M$ be monotone and conservative with a convex domain. Having chosen $x_{0}, \ldots, x_{n} \in \operatorname{dom} M$, the closed polyline $\pi$ whose vertices are $x_{i}$ will be included in the convex set $\operatorname{dom} M$. By virtue of the equality in Theorem 3.12 and the vanishing of the circuital integral of $M$ along $\pi$ we have

$$
\sum_{i=0}^{n}\left\langle x_{i}^{*}, x_{i+1}-x_{i}\right\rangle \leq 0, \quad \text { with } n+1 \equiv 0,
$$

for every choice of $x_{i}^{*} \in M\left(x_{i}\right)$; hence, $M$ is cyclically monotone.

THEOREM 4.7. Conservativity of the inverse map. If $G \subseteq X \times X^{\prime}$ is a monotone graph and the map $\vec{G}$ is conservative with a convex domain, the map $\stackrel{\leftarrow}{G}$ is conservative too.

Proof. By virtue of Remark 4.5 and Theorem 4.6 we have

$$
\left\{\begin{array}{l}
\vec{G} \text { conservative } \\
\operatorname{dom} \vec{G} \text { convex }
\end{array} \Rightarrow \vec{G} \text { cycl. monot. } \Leftrightarrow \overleftarrow{G} \text { cycl. monot: } \Rightarrow \overleftarrow{G}\right. \text { conserv. }
$$

Now let us state the following definitions, which will be useful in the sequel.

Definition 4.8. Conservative monotone graphs. A monotone graph $G \subseteq X \times X^{\prime}$ is said to be conservative if $\operatorname{dom} \vec{G}$ and $\operatorname{dom} \overleftrightarrow{G}$ are convex sets and the maps $\vec{G}$ and $\overleftarrow{G}$ are conservative. Let us remark that, by Theorem 4.7 , it is sufficient to assess the conservativity of one of the two maps $\vec{G}$ or $\overleftarrow{G}$.

Definition 4.9. A set $S \subseteq X$ is said to be $\pi$-connected if for every pair $x, y \in S$ there exists a polyline $\pi \subseteq S$ starting at $x$ and ending at $y$.

Definition 4.10. A set $S \subseteq X$ is said to be quasi-convex if there exists a nonempty convex open set $A \subseteq X$ such that $A \subseteq S \subseteq \bar{A}$, where $\bar{A}$ denotes the closure of $A$. 
In Banach spaces, the domain of a maximal monotone operator is quasi-convex if its convex hull has a nonempty interior [10].

It is worth noting that a convex set with nonempty interior is quasi-convex and that a quasi-convex set is $\pi$-connected.

The concepts introduced so far allow us to state the following.

THEOREM 4.11. The potential of a multivalued map. To any conservative monotone multivalued map $M: X \mapsto X^{\prime}$ with a $\pi$-connected domain, there corresponds, to within an arbitrary additive constant, a convex potential $f: X \mapsto \Re \cup\{+\infty\}$, which is the restriction on $\operatorname{dom} M$ of a lower semicontinuous proper convex functional. The potential $f$ is assumed to be $+\infty$ outside $\operatorname{dom} M$ and is defined on $\operatorname{dom} M$ by

$$
\begin{aligned}
f(x)-f\left(x_{0}\right) & \stackrel{\text { def }}{=} \int_{\pi}\langle M(z), d z\rangle \\
& =\sup \left\{\sum_{i=0}^{n-1}\left\langle x_{i}^{*}, x_{i+1}-x_{i}\right\rangle+\left\langle x_{n}^{*}, x-x_{n}\right\rangle\right\} \\
& =\inf \left\{\sum_{i=0}^{n-1}\left\langle x_{i+1}^{*}, x_{i+1}-x_{i}\right\rangle+\left\langle x^{*}, x-x_{n}\right\rangle\right\},
\end{aligned}
$$

where $\pi$ is an arbitrary polyline in $\operatorname{dom} M$ starting at $x_{0}$ and ending at $x$, the $\sum$ 's are relative to arbitrary refinements of $\pi$, and the choice of $x_{i}^{*} \in M\left(x_{i}\right)$ is inessential.

Proof. Let us first remark that the definition of potential is well posed since the integral of the map is path independent. Its equivalent expressions in terms of finite sums follow, according to Theorem 3.12 , by integrating along an arbitrary polyline in $\operatorname{dom} M$ starting at $x_{0}$ and ending at $x$.

Finally the formula above reveals that, on $\operatorname{dom} M$, the potential is equal to the lower semicontinuous proper convex functional, which is defined as the pointwise supremum of a family of continuous affine functionals $[3,12]$.

Let us notice that, if $\operatorname{dom} M$ is a closed set, $f$ turns out to be a lower semicontinuous proper convex functional.

REMARK 4.12. If dom $M$ is a convex set then the "sup" and the "inf" in Theorem 4.11 can be evaluated for all $x_{1}, \ldots, x_{n}$ in $\operatorname{dom} M$ with $x_{i}^{*} \in M\left(x_{i}\right)$ or, equivalently, for all $\left(x_{i}, x_{i}^{*}\right)$ with $i=1, \ldots, n$ belonging to graph $(M)$. In fact, in this case, the oriented polyline $\pi$ whose vertices are $x_{0}, x_{1}, \ldots, x_{n}, x$ turns out to be included in the convex set $\operatorname{dom} M$ for every choice of $x_{1}, \ldots, x_{n}$ in $\operatorname{dom} M$.

The next proposition yields a preliminary result that will be referred to in the sequel.

Lemma 4.13. Let $f: X \mapsto \Re \cup\{+\infty\}$ be a convex functional whose domain has a nonempty interior. If $f$ is minorized by an affine functional in the interior of its domain, the same relation holds in the whole space $X$.

Proof. It has to be proved that, given $x^{*} \in X^{\prime}$ and $k \in \Re$ such that

$$
f(x) \geq\left\langle x^{*}, x\right\rangle+k \quad \forall x \in \operatorname{int} \operatorname{dom} f,
$$


the same property holds for every $x \in X$. The inequality trivially holds if $x \notin$ $\operatorname{dom} f$. On the other hand, considering the closed halfspace

$$
S^{+}=\left\{(x, t) \in X \times \Re: t \geq\left\langle x^{*}, x\right\rangle+k\right\}
$$

and the set

$$
\text { int epi } f=\{(x, t) \in X \times \Re: x \in \operatorname{int} \operatorname{dom} f, t>f(x)\},
$$

it follows that int epi $f \subseteq S^{+}$and, hence, trivially, intepi $f \subseteq S^{+}$. In a topological vector space, a convex set with nonempty interior is included in the closure of its interior; then we have

$$
\text { epi } f \subseteq \overline{\text { int epi } f} \subseteq S^{+} \text {. }
$$

So we have

and, by definition of $S^{+}$,

$$
(x, f(x)) \in S^{+} \quad \forall x \in \operatorname{dom} f,
$$

$$
f(x) \geq\left\langle x^{*}, x\right\rangle+k
$$

for every $x \in \operatorname{dom} f$.

THeOREM 4.14. Maximality of the subdifferential of a potential. Let $M: X \mapsto X^{\prime}$ be a conservative monotone map with $\operatorname{dom} M$ quasi-convex, and let $G=\operatorname{graph}(M)$. The graph of the subdifferential $\partial f$ of the potential $f$ of $M$ is the unique maximal monotone extension of $G$ in v-strip $(G)$. Hence, if $M$ is maximal monotone in v-strip $(G)$ then $M=\partial f$.

Proof. To prove the theorem let us first notice that, by Remark 3.10, we have

$$
f(y)-f(x) \stackrel{\text { def }}{=} \int_{x}^{y}\langle M(z), d z\rangle \geq\left\langle x^{*}, y-x\right\rangle \quad \forall\left(x, x^{*}\right) \in G, y \in \operatorname{int} \operatorname{dom} M,
$$

since the line segment connecting $x$ and $y$ lies in the quasi-convex set $\operatorname{dom} M$. By virtue of Lemma 4.13, the inequality above does in fact hold for all $y \in X$; then, by definition, $x^{*} \in \partial f(x)$. It follows that

$$
G \subseteq \operatorname{graph}(\partial f) \subseteq \operatorname{v-strip}(G) .
$$

Since the subdifferentials of convex functionals are monotone maps, the inclusion above simply states that graph $(\partial f)$ is a monotone extension of $G$.

Let us show that the extension is maximal. To this end consider the family $\mathscr{F}_{\text {exv }}$ of the monotone extension $G_{\text {exv }}$ of $G$ in the v-strip $(G)$. By virtue of Remarks 3.13 and 4.2, the potential $f$ of $M$ will be also the potential of the right map associated with any graph of $\mathscr{F}_{\text {exv }}$ so that, being graph $(\partial f) \in \mathscr{F}_{\text {exv }}$ and $G_{\text {exv }} \subseteq \operatorname{graph}(\partial f)$ for any $G_{\text {exv }} \in \mathscr{F}_{\text {exv }}$, graph $(\partial f)$ turns out to be the unique maximal element of the family.

REMARK 4.15. A simpler proof of Theorem 4.14 can be carried out by assuming the convexity of dom $M$ instead of its quasi-convexity. In fact, under this hypothesis, the inequality appearing in the proof of the theorem does hold for every $y \in \operatorname{dom} M$ and then trivially for every $y \in X$.

The next theorem provides the generalization to the present context of a classical result of integral calculus: the potential of an operator, which is the gradient of a functional coincides, to within an additive constant, with the functional itself. 
Theorem 4.16. The integral theorem. Let $g: X \mapsto \Re \cup\{+\infty\}$ be a proper convex functional, continuous in the nonempty set int dom $g$. The subdifferential $\partial g: X \mapsto$ $X^{\prime}$ is a conservative monotone multivalued map, and the restriction of $g$ to $\operatorname{dom} \partial g$ is the potential of $\partial g$, to within an arbitrary additive constant.

Proof. It is well known that $\partial g$ turns out to be cyclically monotone $[9,11,12]$ and, hence, by Theorem 4.6, monotone and conservative. Since $g$ is continuous in int dom $g$, we have [8] int dom $g \subseteq \operatorname{dom} \partial g$.

Let $x_{0} \in$ int dom $g$. For all $x \in \operatorname{dom} \partial g \subseteq \operatorname{dom} g$, the line segment starting at $x_{0}$ and ending at $x$ belongs to int dom $g$ (except at most the point $x$ ).

Denoting by $f$ the potential of $\partial g$, we have

$$
\begin{aligned}
f(x)-f\left(x_{0}\right) & \stackrel{\text { def }}{=} \int_{x_{0}}^{x}\langle\partial g(z), d z\rangle \\
& =\int_{0}^{1}\langle\partial g(\hat{x}(t)), h\rangle d t=\int_{0}^{1} \partial \psi(t) d t \\
& =\int_{0}^{1} d^{+} \psi(t) d t=\int_{0}^{1} d^{-} \psi(t) d t \\
& =g(x)-g\left(x_{0}\right),
\end{aligned}
$$

where $\hat{x}(t)=x_{0}+t h$ with $h=x-x_{0}, t \in[0,1], \psi(t)=g(\hat{x}(t)), d^{+} \psi$ and $d^{-} \psi$ are respectively the right and the left derivative of $\psi(t)$, and the equality $\left[d^{-} \psi(t), d^{+} \psi(t)\right]=\partial \psi(t)=\langle\partial g(\hat{x}(t)), h\rangle, t \in[0,1[$, follows from the continuity of $g$.

Corollary 4.17. Maximality of the subdifferential of a convex functional. If $g: X$ $\mapsto \Re \cup\{+\infty\}$ is a proper convex functional, continuous in the nonempty set int dom $g$, then the subdifferential $\partial g$ is maximal monotone in v-strip of $\operatorname{graph}(\partial g)$.

Proof. Let $f: X \mapsto \Re \cup\{+\infty\}$ be the potential of $\partial g$. It is obvious that the map $\partial g$ is conservative and that $\operatorname{dom} \partial g$ is quasi-convex. Theorem 4.14 ensures then that $\partial f$ is the unique maximal monotone extension of $\partial g$ in v-strip $(\partial g)$. Being $\operatorname{dom} \partial f=\operatorname{dom} \partial g$, the theorem will be proved if we show that $\operatorname{graph}(\partial f) \subseteq$ $\operatorname{graph}(\partial g)$. To this end we observe that

$$
\left(x, x^{*}\right) \in \operatorname{graph}(\partial f) \stackrel{\text { def }}{\Longleftrightarrow} f(y)-f(x) \geq\left\langle x^{*}, y-x\right\rangle \quad \forall y \in \operatorname{dom} f \supseteq \operatorname{int} \operatorname{dom} g .
$$

Since, by Theorem 4.16, $f=g$ on $\operatorname{dom} \partial g$, we get

$$
g(y)-g(x) \geq\left\langle x^{*}, y-x\right\rangle \quad \forall y \in \text { int dom } g .
$$

Then, by virtue of Lemma 4.13 , the inequality above holds $\forall y \in X$ so that $\left(x, x^{*}\right) \in$ $\operatorname{graph}(\partial g)$.

Incidentally, we note that a convex functional $f: \Re^{n} \mapsto \Re \cup\{+\infty\}$ is continuous in the interior of its domain.

The following theorem yields a basic result of the potential theory for conservative monotone graphs (see Definition 4.8).

THEOREM 4.18. Complementary potentials. With any conservative monotone graph $G \subseteq X \times X^{\prime}$ there can be associated a pair of proper convex functionals $f, f_{c}$, called 
the complementary potentials of $G$, which meet the relation

$$
\left[f(x)-f\left(x_{0}\right)\right]+\left[f_{c}\left(x^{*}\right)-f_{c}\left(x_{0}^{*}\right)\right]=\left\langle x^{*}, x\right\rangle-\left\langle x_{0}^{*}, x_{0}\right\rangle
$$

for all $\left(x, x^{*}\right),\left(x_{0}, x_{0}^{*}\right) \in G$.

Proof. By virtue of Theorem 4.11 the potential $f_{c}$ associated with $\overleftarrow{G}$ is given on $\operatorname{dom} \stackrel{\leftarrow}{G}$ by

$$
\begin{aligned}
f_{c}\left(x^{*}\right)-f_{c}\left(x_{0}^{*}\right) & \stackrel{\text { def }}{=} \int_{\pi^{*}}\left\langle\overleftarrow{G}\left(z^{*}\right), d z^{*}\right\rangle \\
& =\inf \left\{\sum_{i=0}^{n-1}\left\langle x_{i+1}^{*}-x_{i}^{*}, x_{i+1}\right\rangle+\left\langle x^{*}-x_{n}^{*}, x\right\rangle\right\},
\end{aligned}
$$

where $\pi^{*} \subseteq \operatorname{dom} \overleftarrow{G}$ is a polyline starting at $x_{0}^{*}$ and ending at $x^{*}$

The complementarity relation can then be proved by a suitable rearrangement of the terms in the $\sum$ 's defining the potential $f$ of $\vec{G}$ and observing that the "sup" and the "inf" appearing in the next formulas can be evaluated, by virtue of Remark 4.12 , for every choice of an $n$-tuple $\left(x_{i}^{*}, x_{i}\right)$ belonging to $G$. Then we have

$$
\begin{aligned}
f(x)-f\left(x_{0}\right) & =\sup \left\{\sum_{i=0}^{n-1}\left\langle x_{i}^{*}, x_{i+1}-x_{i}\right\rangle+\left\langle x_{n}^{*}, x-x_{n}\right\rangle\right\} \\
& =\sup \left\{\sum_{i=0}^{n-1}\left\langle x_{i}^{*}-x_{i+1}^{*}, x_{i+1}\right\rangle+\left\langle x_{n}^{*}-x^{*}, x\right\rangle\right\}+\left\langle x^{*}, x\right\rangle-\left\langle x_{0}^{*}, x_{0}\right\rangle \\
& =-\inf \left\{\sum_{i=0}^{n-1}\left\langle x_{i+1}^{*}-x_{i}^{*}, x_{i+1}\right\rangle+\left\langle x^{*}-x_{n}^{*}, x\right\rangle\right\}+\left\langle x^{*}, x\right\rangle-\left\langle x_{0}^{*}, x_{0}\right\rangle \\
& =f_{c}\left(x_{0}^{*}\right)-f_{c}\left(x^{*}\right)+\left\langle x^{*}, x\right\rangle-\left\langle x_{0}^{*}, x_{0}\right\rangle,
\end{aligned}
$$

where $\left(x, x^{*}\right) \in G$ and $\left(x_{0}, x_{0}^{*}\right) \in G$.

THEOREM 4.19. Maximality in the frame. If $G \subseteq X \times X^{\prime}$ is a conservative monotone graph, the set $G_{m}=\operatorname{graph}(\partial f) \cap \operatorname{graph}\left(\partial f_{c}\right)$ is the unique maximal monotone extension of $G$ in frame $(G)$.

Proof. Let us consider the family $\mathscr{F}_{\text {ex }}$ of the monotone extensions $G_{\text {ex }}$ of $G$ in frame $(G)$. Each element $G_{\text {ex }}$ of this family trivially belongs to both families $\mathscr{F}_{\text {exv }}$ and $\mathscr{F}_{\text {exh }}$ of the monotone extensions of $G$ in v-strip $(G)$ and h-strip $(G)$ respectively. Hence it will be included in the maximal elements of $\mathscr{F}_{\text {exv }}$ and $\mathscr{F}_{\text {exh }}$. By Remark 4.15, it follows that

$$
G_{\text {ex }} \subseteq \operatorname{graph}(\partial f) \cap \operatorname{graph}\left(\partial f_{c}\right) \quad \forall G_{\text {ex }} \in \mathscr{F}_{\text {ex }} .
$$

Then the set graph $(\partial f) \cap \operatorname{graph}\left(\partial f_{c}\right)$, being itself an element of $\mathscr{F}_{\text {ex }}$, will be the unique maximal element of the family.

An immediate consequence of the previous theorem is

REMARK 4.20. If $G$ is a conservative monotone graph, the complementarity relation between $f$ and $f_{c}$ does hold for all the pairs $\left(x, x^{*}\right)$ which belong to the maximal monotone extension $G_{m}$ of $G$ in frame $(G)$. 
Further from Theorem 4.19 we infer that

REMARK 4.21. If $G \subseteq X \times X^{\prime}$ is a conservative maximal monotone graph then $G=\operatorname{graph}(\partial f)=\operatorname{graph}\left(\partial f_{c}\right)$.

It is worth noting that graph $(\partial f)$ and graph $\left(\partial f_{c}\right)$ are not necessarily coincident unless $G$ is a conservative maximal monotone graph.

An illustrative example is provided in Fig. 3 where: (a) is the sketch of a graph $G$, which is maximal in its frame, and $\left(b_{1}\right)-\left(b_{2}\right),\left(c_{1}\right)-\left(c_{2}\right)$ respectively show
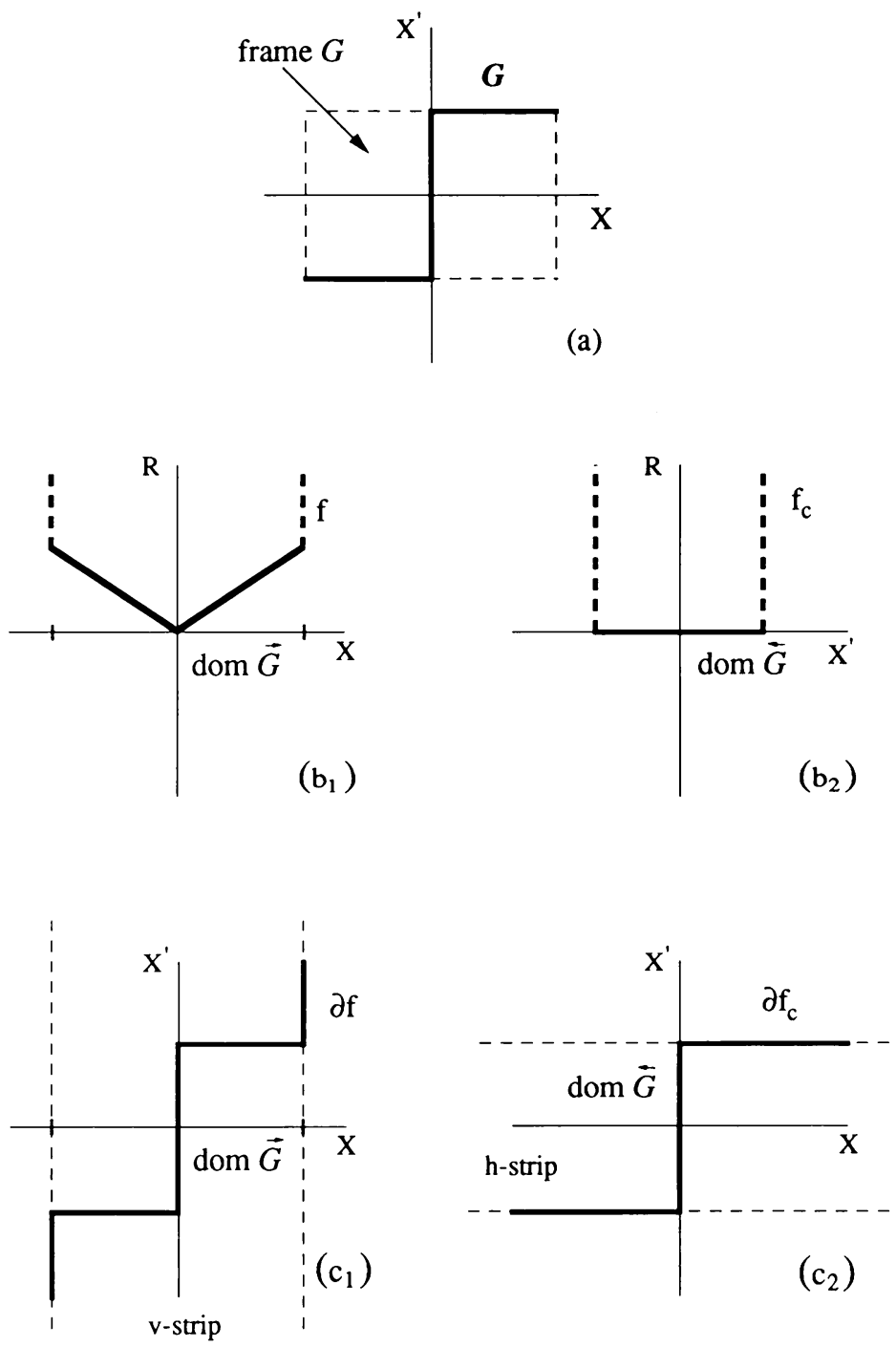

FIG. 3. A monotone graph with its potentials and the corresponding subdifferentials maps. 
the complementary potentials associated with $G$ and the graphs of the corresponding subdifferential maps.

The complementarity property of the convex potentials $f$ and $f_{c}$ associated with a conservative monotone graph $G \subseteq X \times X^{\prime}$ can equivalently be formulated by stating that the functional

$$
I\left(x, x^{*}\right) \stackrel{\text { def }}{=} f(x)+f_{c}\left(x^{*}\right)-\left\langle x^{*}, x\right\rangle
$$

is finite and constant on the maximal monotone extension $G_{m}$ of $G$ in frame $(G)$.

An important minimality property for $I\left(x, x^{*}\right)$ is provided by the next theorem.

THEOREM 4.22. Minimality of the invariant. Let $G \subseteq X \times X^{\prime}$ be a conservative monotone graph and $f, f_{c}$ the associated complementary potentials. The finite constant value taken by $I\left(x, x^{*}\right)$ on $G_{m}=\operatorname{graph}(\partial f) \cap \operatorname{graph}\left(\partial f_{c}\right)$ is an absolute minimum in the product space $X \times X^{\prime}$. Denoting by

$$
\mathscr{M}=\left\{\left(\bar{x}, \bar{x}^{*}\right) \in X \times X^{\prime} \mid I\left(\bar{x}, \bar{x}^{*}\right)=\min I\right\},
$$

the minimal set of $I\left(x, x^{*}\right)$, we have

$$
\mathscr{M}=G_{m} .
$$

Proof. Let us first prove that $\operatorname{graph}(\partial f) \cap \operatorname{graph}\left(\partial f_{c}\right) \subseteq \mathscr{M}$. In fact, if $\left(x, x^{*}\right) \in$ $G_{m}$, we have by definition

$$
\begin{aligned}
f(y)-f(x) & \geq\left\langle x^{*}, y-x\right) \quad \forall y \in X, \\
f_{c}\left(z^{*}\right)-f_{c}\left(x^{*}\right) & \geq\left\langle z^{*}-x^{*}, x\right\rangle \quad \forall z^{*} \in X^{\prime} .
\end{aligned}
$$

Adding $f_{c}\left(x^{*}\right)$ and $f(x)$ respectively to both members of the first and the second inequality, we get

$$
\begin{array}{ll}
I\left(y, x^{*}\right) \geq I\left(x, x^{*}\right) & \forall y \in X, \\
I\left(x, z^{*}\right) \geq I\left(x, x^{*}\right) & \forall z^{*} \in X^{\prime} .
\end{array}
$$

Now, for every $y \in \operatorname{dom} \vec{G}_{m}$, there exists a $y^{*} \in X^{\prime}$ such that $\left(y, y^{*}\right) \in G_{m}$. Then from the latter inequality we get

$$
I\left(y, z^{*}\right) \geq I\left(y, y^{*}\right)=I\left(x, x^{*}\right) \quad \forall z^{*} \in X^{\prime} .
$$

Since $I\left(y, z^{*}\right)=+\infty$ when $y \notin \operatorname{dom} \vec{G}_{m}$, it turns out that

$$
I\left(y, z^{*}\right) \geq I\left(x, x^{*}\right) \quad \forall y \in X, \forall z^{*} \in X^{\prime},
$$

which implies that $\left(x, x^{*}\right) \in \mathscr{M}$.

The opposite inclusion $\mathscr{M} \subseteq \operatorname{graph}(\partial f) \cap \operatorname{graph}\left(\partial f_{c}\right)$ follows by observing that, if $\left(x, x^{*}\right) \in \mathscr{M}$, by definition we have

$$
I\left(x, x^{*}\right) \leq I\left(y, z^{*}\right) \quad \forall y \in X, \forall z^{*} \in X^{\prime}
$$

and then "a fortiori"

$$
\begin{array}{ll}
I\left(y, x^{*}\right) \geq I\left(x, x^{*}\right) & \forall y \in X, \\
I\left(x, z^{*}\right) \geq I\left(x, x^{*}\right) & \forall z^{*} \in X^{\prime},
\end{array}
$$


from which the result is obtained by reverting the first step in the proof of the theorem.

Without loss in generality we can assume that $I\left(x, x^{*}\right)=0$ on $G$ so that the minimality property yields

$$
f_{c}\left(x^{*}\right) \geq\left\langle x^{*}, x\right\rangle-f(x) \quad \forall\left(x, x^{*}\right) \in X \times X^{\prime} .
$$

A comparison with Fenchel's inequality:

$$
f^{*}\left(x^{*}\right) \geq\left\langle x^{*}, x\right\rangle-f(x) \quad \forall\left(x, x^{*}\right) \in X \times X^{\prime}
$$

could lead at first sight to the erroneous conclusion that, for any conservative monotone graph, $f_{c}=f^{*}$ must result on the whole $X^{\prime}$.

In fact, Fenchel's inequality is optimal since, by definition, $f^{*}\left(x^{*}\right)$ is the least upper bound of the numerical set described by the right-hand side when $x$ ranges over $X$. When $x^{*} \in \operatorname{dom} \overleftarrow{G}$, the values of the potential $f_{c}$ and of the functional $f^{*}$ are both equal to the maximum of this numerical set, so that

$$
f_{c}\left(x^{*}\right)=\max \left\{\left\langle x^{*}, x\right\rangle-f(x) \mid x \in X\right\}=f^{*}\left(x^{*}\right) \quad \forall x^{*} \in \operatorname{dom} \overleftarrow{G}
$$

On the contrary, outside $\operatorname{dom} \stackrel{\leftarrow}{G}$, we can just state $f_{c}\left(x^{*}\right) \geq f^{*}\left(x^{*}\right)$ since by definition $f_{c}\left(x^{*}\right)=+\infty$ while $f^{*}\left(x^{*}\right)$ could assume a finite value.

The failure of the identity $f_{c}=f^{*}$ is depicted by the example in Fig. 4 where the potentials $f$ and $f_{c}$ of the graph reported in Fig. 3 and the corresponding Fenchel's conjugates are sketched.
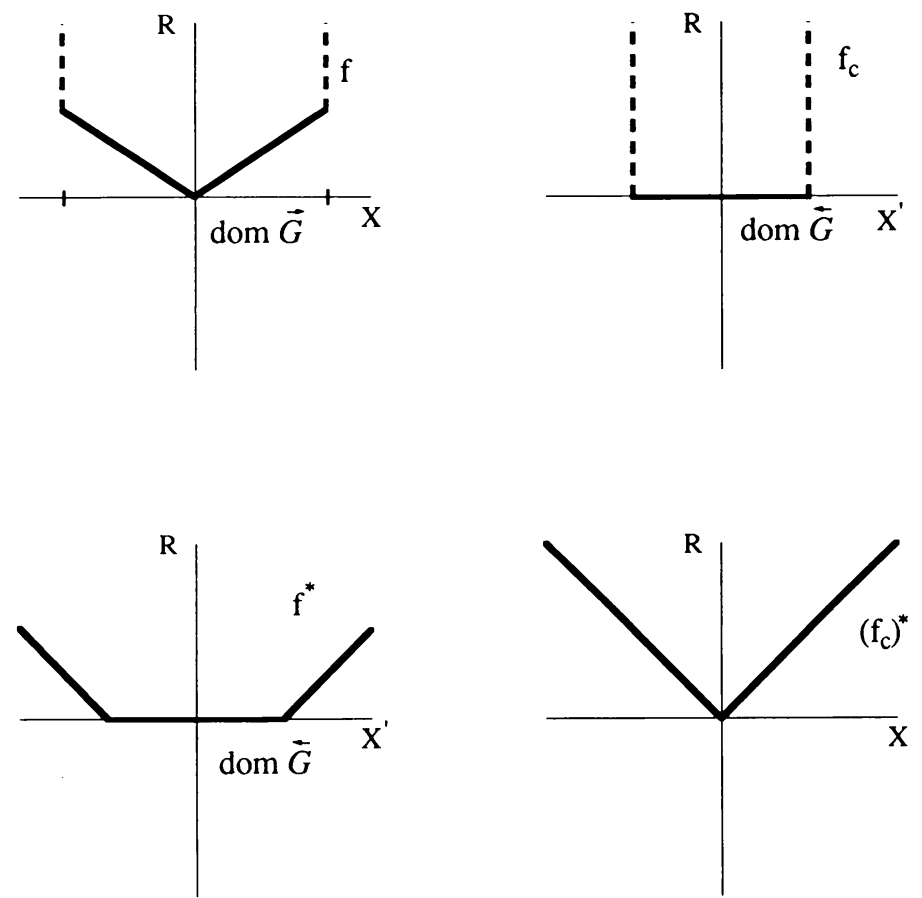

Fig. 4. Fenchel's conjugates of the potentials $f$ and $f_{c}$ of the graph $G$ in Fig. 3. 

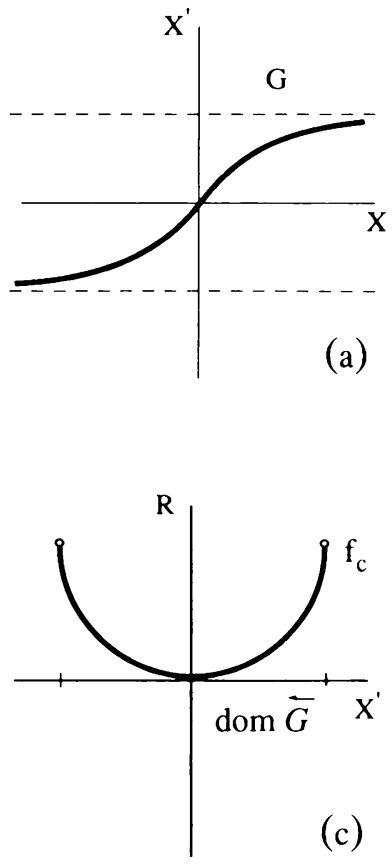
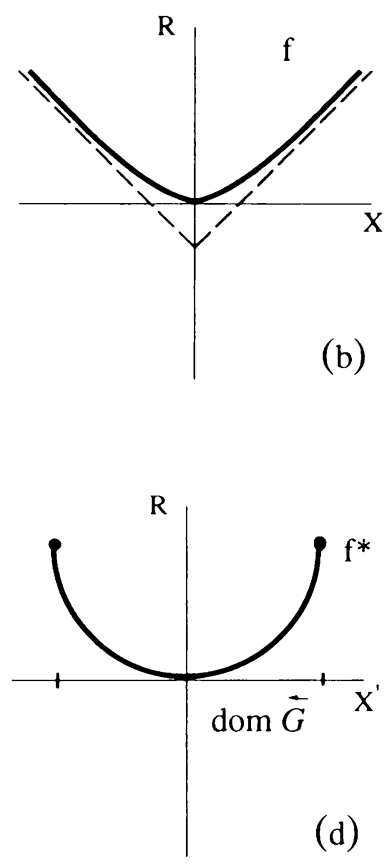

Fig. 5. Maximal monotone graph $G$ for which $f^{*} \neq f_{c}$.

The identity $f_{c}=f^{*}$ fails even when the graph $G$ is maximal monotone as depicted in Fig. 5. Nevertheless this identity can be stated under a stronger hypothesis as shown by the following theorem.

THEOREM 4.23. Conjugacy property of complementary potentials. Let $G \subseteq X \times X^{\prime}$ be a conservative maximal monotone graph, $f$ and $f_{c}$ the complementary potentials associated with $G$, and $f^{*},\left(f_{c}\right)^{*}$ the corresponding Fenchel's conjugates. If at the point $x^{*} \in X^{\prime}$ the subdifferential of $f^{*}$ is a nonempty set, we have $f_{c}\left(x^{*}\right)=f^{*}\left(x^{*}\right)$. Hence, if $f^{*}$ is everywhere subdifferentiable in its domain, it turns out that $f_{c}=f^{*}$. An analogous statement can be formulated in terms of $f$ and $\left(f_{c}\right)^{*}$.

Proof. Since $G$ is maximal monotone in v-strip $(G)$, Remark 4.15 implies that $G=\operatorname{graph}(\partial f)$. It is well known that [12] $\operatorname{graph}(\partial f) \subseteq \operatorname{graph}\left(\partial f^{*}\right)$ so that, by the maximality of $G=\operatorname{graph}(\partial f)$, we have

$$
\operatorname{graph}(\partial f)=\operatorname{graph}\left(\partial f^{*}\right)
$$

Then, it follows that the Fenchel's equality

$$
f(x)+f^{*}\left(x^{*}\right)-\left\langle x^{*}, x\right\rangle=0
$$

holds for each pair $\left(x, x^{*}\right) \in G$. By subtracting the equality above from the definition of $I\left(x, x^{*}\right)$ we get

$$
f_{c}\left(x^{*}\right)=f^{*}\left(x^{*}\right) \quad \forall x^{*} \in \operatorname{dom}\left(\partial f^{*}\right),
$$

and the result is proved. A dual argument gives the last part of the statement. 
Acknowledgment. The financial support of the Italian Ministry for Scientific and Technological Research is gratefully acknowledged.

\section{REFERENCES}

[1] J. P. Aubin and I. Ekeland, Applied Nonlinear Analysis, John Wiley and Sons, New York, Chichester, Brisbane, Toronto, and Singapore, 1984

[2] N. Bourbaki, Fonctions d'une variable réelle, Hermann, Paris, 1976

[3] I. Ekeland and R. Temam, Analyse convexe et problemes variationnels, Dunod, Paris, Bruxelles, and Montreal, 1974

[4] W. Fenchel, On conjugate convex functions, Canad. J. Math. 1, 73-77 (1949)

[5] A. D. Ioffe and V. M. Tihomirov, The theory of extremal problems, Nauka, Moscow, 1979, English transl., North-Holland, Amsterdam, 1979

[6] A. N. Kolmogorov and S. V. Fomin, Elements of the theory of functions and functional analysis, Graylock Press, Baltimore, MD, 1961

[7] G. J. Minty, On the monotonicity of the gradient of a convex function, Pacific J. Math. 14, 243-247 (1964)

[8] J. J. Moreau, Fonctionelles convexes, lecture notes, séminaire: équationes aux dérivées partielles, Collègie de France, 1966

[9] R. T. Rockafellar, Characterization of the subdifferentials of convex functions, Pacific J. Math. 17, 497-510 (1966); A correction to the maximality proof given in this paper appears in [11].

[10] R. T. Rockafellar, Local boundedness of nonlinear, monotone operators, Michigan Math. J. 16, 397-407 (1969)

[11] R. T. Rockafellar, On the maximal monotonicity of subdifferential mappings, Pacific J. Math. 33, 209-216 (1970)

[12] R. T. Rockafellar, Convex Analysis, Princeton Univ. Press, Princeton, NJ, 1970

[13] M. M. Vainberg, Variational methods for the study of nonlinear operators, Holden-Day, Inc., San Francisco, CA, 1964

[14] K. Yosida, Functional Analysis, 6th ed., Springer-Verlag, Berlin, 1980 